Turk. J. Math. Comput. Sci.

13(1)(2021) 135-144

(C) MatDer

DOI : $10.47000 /$ tjmcs. 877094

\title{
Quasi-Hemi-Slant Conformal Submersions from Almost Hermitian Manifolds
}

\author{
ŞENER Yanan \\ Department of Mathematics, Faculty of Arts and Science, Adlyaman University, 02040, Adiyaman, Turkey.
}

Received: 08-02-2021 • Accepted: 03-05-2021

\begin{abstract}
AвSTRACT. In this study, we introduce some geometric properties of quasi-hemi-slant conformal submersions from an almost Hermitian manifold to a Riemannian manifold. We give an explicit example for this type submersions and obtain integrability conditions for certain distributions. Lastly, we search totally geodesicity on base manifold of the map.
\end{abstract}

2010 AMS Classification: 53C55, 53C15

Keywords: Conformal submersion, quasi-hemi-slant conformal submersion.

\section{INTRODUCTION}

The theory of Riemannian submersions between Riemannian manifolds was initially studied by O'Neill [12] and Gray [7]. Then, this theory was expansed to almost Hermitian submersions between almost Hermitian manifolds [23]. After these studies, this theory was widely studied in $[6,19]$. Şahin defined various types of Riemannian submersions from an almost Hermitian manifold onto a Riemannian manifold such as anti-invariant submersions [15], semi-invariant submersions [17] and slant submersions [16], see also [9,13,20]. Also, Şahin gave some main results about Riemannian submersions and an application on robotic theory [18]. Therefore, a new vision on submersions by applying conformality conditions was presented by Akyol and Şahin [2]- [4], see also [8, 14]. Riemannian submersions have many applications as texture mapping, remeshing and simulation [10], computer graphics and medical imaging fields [21], brain mapping research [22].

In this study, in Section 2, we give some basic notions to be used along this study. In Section 3, we define quasi-hemislant conformal submersion from an almost Hermitian manifold onto a Riemannian manifold which is the expansion of conformal semi-slant submersions [1], conformal semi-invariant submersions [3], conformal hemi-slant submersions [8]. We introduce some geometric properties for quasi-hemi-slant conformal submersions. In Section 4, we give some conditions for certain distributions to define totally geodesic foliation on base manifold.

Email addresses: syanan@adiyaman.edu.tr (Ş. Yanan) 


\section{Preliminaries}

In this section, we give several definitions and results to be used throughout the study for quasi-hemi-slant conformal Riemannian submersions.

An even-dimensional Riemannian manifold $\left(M, g_{M}, J\right)$ is called an almost Hermitian manifold if there exists a tensor field $J$ of type $(1,1)$ on $M$ such that $J^{2}=-I$ where $I$ denotes the identity transformation of $T M$ and

$$
g_{M}(X, Y)=g_{M}(J X, J Y), \forall X, Y \in \Gamma(T M) .
$$

Let $\left(M, g_{M}, J\right)$ be an almost Hermitian manifold and its Levi-Civita connection is $\nabla$ with respect to $g_{M}$. If $J$ is parallel with respect to $\nabla$, i.e.

$$
\left(\nabla_{X} J\right) Y=0
$$

we say $M$ is a Kähler manifold [24].

Let $\Phi:\left(M, g_{M}\right) \longrightarrow\left(N, g_{N}\right)$ be a smooth map between Riemannian manifolds. The second fundamental form of $\Phi$ is defined by

$$
\left(\nabla \Phi_{*}\right)(X, Y)=\stackrel{N}{\nabla_{X}^{\Phi}} \Phi_{*}(Y)-\Phi_{*}\left(\stackrel{M}{\nabla}{ }_{X} Y\right)
$$

for $X, Y \in \Gamma(T M)$. The second fundamental form $\nabla \Phi_{*}$ is symmetric [11]. Here, $\Phi_{*}$ is differential map of $\Phi$ from tangent space of $M$ at a point $x \in M$ to tangent space of $N$ at $\Phi(x)$ such that $\Phi_{*}: T_{x} M \longrightarrow T_{\Phi(x)} N$.

A smooth map $\Phi:\left(M^{m}, g_{M}\right) \longrightarrow\left(N^{n}, g_{N}\right)$ between Riemannian manifolds is called a Riemannian submersion if $\Phi$ has maximal rank and the differential $\Phi_{*}$ preserves the lengths of horizontal vectors. On the other hand, let $\Phi:\left(M, g_{M}\right) \longrightarrow\left(N, g_{N}\right)$ be a smooth map between Riemannian manifolds and $p \in M$. Then, $\Phi$ is called horizontally weakly conformal at $p$ if either (i) $\Phi_{* p}=0$ or (ii) $\Phi_{* p}$ is surjective and there exists a number $\wedge(p) \neq 0$ such that

$$
g_{N}\left(\Phi_{* p}(X), \Phi_{* p}(Y)\right)=\wedge(p) g_{M}(X, Y)
$$

for $X, Y \in \Gamma\left(\left(\operatorname{ker} \Phi_{*}\right)^{\perp}\right)$. We call the point $p$ is of type (i) as a critical point and we shall call the point $\mathrm{p}$ a regular point if it satisfied the type (ii). At a critical point, $\operatorname{rank}\left(\Phi_{* p}\right)=0$, at a regular point, $\Phi_{* p}$ has $\operatorname{rank} n$ and $\Phi$ is a submersion. Additionally, the positive number $\wedge(p)$ is called the square dilation of $\Phi$ at $p$. The map $\Phi$ is called horizontally weakly conformal or semi conformal on $M$ if it is horizontally weakly conformal at every point of $M$ and it has no critical point, then we call it as a horizontally conformal submersion [5].

If a vector field $X$ on $M$ is related to a vector field $X^{\prime}$ on $N$, we say $X$ is a projectable vector field. If $X$ is both a horizontal and a projectable vector field, we say $X$ is a basic vector field on $M$. From now on, when we mention a horizontal vector field, we always consider a basic vector field [5].

Then, O'Neill's tensor fields $T$ and $A$ for Riemannian submersions are defined as

$$
\begin{aligned}
A_{X} Y & =\stackrel{M}{h} \nabla_{h X} v Y+v \nabla_{h X} h Y, \\
T_{X} Y & =\stackrel{M}{\nabla_{v X} v Y+v} \stackrel{M}{\nabla_{v X}} h Y,
\end{aligned}
$$

for $X, Y \in \Gamma(T M)$ with the Levi-Civita connection $\stackrel{M}{\nabla}$ of $g_{M}$. For any $X \in \Gamma(T M), T_{X}$ and $A_{X}$ are skew-symmetric operators on $\left(\Gamma(T M), g\right.$ ) reversing the horizontal and the vertical distributions. Also, $T$ is vertical, $T_{X}=T_{v X}$, and $A$ is horizontal, $A_{X}=A_{h X}$. Note that the tensor field $T$ is symmetric on the vertical distribution [12]. In addition, from (2.4) and (2.5) we have

$$
\begin{aligned}
& \stackrel{M}{\nabla}_{U} V=T_{U} V+\hat{\nabla}_{U} V, \\
& \stackrel{M}{\nabla}_{U} X=h \nabla_{U} X+T_{U} X, \\
& \stackrel{M}{\nabla}_{X} V=A_{X} V+\nu \nabla_{X} V, \\
& \stackrel{M}{\nabla}_{X} Y=h{ }^{M} Y+A_{X} Y
\end{aligned}
$$

for $X, Y \in \Gamma\left(\left(\operatorname{ker} \Phi_{*}\right)^{\perp}\right)$ and $U, V \in \Gamma\left(\operatorname{ker} \Phi_{*}\right)$, where $\hat{\nabla}_{U} V=\nu \stackrel{M}{\nabla_{U}} V[6]$.

From [5], we have the following lemma. 
Lemma 2.1. Suppose that $\Phi:\left(M^{m}, g_{M}\right) \longrightarrow\left(N^{n}, g_{N}\right)$ is a horizontally conformal submersion. Then, we have

$$
\begin{aligned}
& \left(\nabla \Phi_{*}\right)(X, Y)=X(\ln \lambda) \Phi_{*}(Y)+Y(\ln \lambda) \Phi_{*}(X)-g_{M}(X, Y) \Phi_{*}(\operatorname{grad}(\ln \lambda)), \\
& \left(\nabla \Phi_{*}\right)(U, V)=-\Phi_{*}\left(T_{U} V\right) \\
& \left(\nabla \Phi_{*}\right)(X, V)=-\Phi_{*}\left(A_{X} V\right) .
\end{aligned}
$$

for any horizontal vector fields $X, Y$ and vertical vector fields $U, V$ [5]. Here, $\lambda$ is the dilation of $\Phi$ at a point $x \in M$ and it is a continuous function as $\lambda: M \longrightarrow[0, \infty)$.

\section{Quasi-Hemi-Slant Conformal Riemannian Submersions}

Firstly, we give definition of quasi-hemi-slant Riemannian submersions from almost Hermitian manifolds to Riemannian manifolds.

Definition 3.1. Let $\Phi:\left(M, g_{M}, J\right) \longrightarrow\left(N, g_{N}\right)$ be a conformal submersion such that its vertical distribution $\operatorname{ker} \Phi_{*}$ admits three orthogonal distributions $D, D_{\theta}$ and $D_{\perp}$ which are invariant $(J(D)=D)$, slant (the angle $\theta$ between $D_{\theta}$ and $J\left(D_{\theta}\right)$ is a constant $)$ and anti-invariant $\left(J\left(D_{\perp}\right) \subseteq\left(k e r \Phi_{*}\right)^{\perp}\right)$, respectively, i.e.

$$
\operatorname{ker} \Phi_{*}=D \oplus D_{\theta} \oplus D_{\perp} .
$$

Then, we say $\Phi$ is a quasi-hemi-slant conformal submersion and the angle $\theta$ is called the quasi-hemi-slant angle of the map.

Here, we have some particular cases;

i) If the distribution $D=\{0\}$ then the map $\Phi$ is a conformal hemi-slant submersion [8].

ii) If the distribution $D_{\theta}=\{0\}$ then the map $\Phi$ is a conformal semi-invariant submersion [3].

iii) If the distribution $D_{\perp}=\{0\}$ then the map $\Phi$ is a conformal semi-slant submersion [1].

Hence, quasi-hemi-slant conformal submersions are generalization of conformal hemi-slant submersions, conformal semi-invariant submersions and conformal semi-slant submersions.

Let $\Phi:\left(M, g_{M}, J\right) \longrightarrow\left(N, g_{N}\right)$ be a quasi-hemi-slant conformal submersion. Then we have

$$
T M=\operatorname{ker} \Phi_{*} \oplus\left(\operatorname{ker} \Phi_{*}\right)^{\perp} .
$$

A vertical vector field $U$ can written as

$$
U=\tilde{P} U+\tilde{Q} U+\tilde{R} U
$$

where $\tilde{P}, \tilde{Q}$ and $\tilde{R}$ are projections onto $D, D_{\theta}$ and $D_{\perp}$, respectively. We get

$$
J U=\phi U+\psi U
$$

where $\phi U \in \Gamma\left(k e r \Phi_{*}\right)$ and $\psi U \in \Gamma\left(\left(k e r \Phi_{*}\right)^{\perp}\right)$. From (3.3), (3.4) and Definition 3.1, we obtain $\psi \tilde{P} U=0, \phi \tilde{R} U=0$ and

$$
J U=\phi \tilde{P} U+\phi \tilde{Q} U+\psi \tilde{Q} U+\psi \tilde{R} X
$$

Hence, we can write

$$
J\left(k e r \Phi_{*}\right)=D \oplus \phi D_{\theta} \oplus \psi D_{\theta} \oplus J\left(D_{\perp}\right) .
$$

Using (3.6), we have

$$
\left(k e r \Phi_{*}\right)^{\perp}=\psi D_{\theta} \oplus J\left(D_{\perp}\right) \oplus \mu
$$

where $\mu$ is the orthogonal complement distributions of $\psi D_{\theta} \oplus J\left(D_{\perp}\right)$ in $\left(\operatorname{ker} \Phi_{*}\right)^{\perp}$ and $\mu$ is the invariant with respect to $J$. Lastly, for a horizontal vector field $X$, we have

$$
J X=B X+C X
$$

where $B X \in \Gamma\left(\psi D_{\theta} \oplus J\left(D_{\perp}\right)\right)$ and $C X \in \Gamma(\mu)$.

Here that one can easily see from (3.1) - (3.7);

$$
\begin{array}{r}
\phi D_{\theta}=D_{\theta}, \quad \phi D_{\perp}=\{0\}, \quad B \psi D_{\theta}=D_{\theta}, \quad B \psi D_{\perp}=D_{\perp}, \quad \psi D=\{0\}, \\
\phi^{2}+B \psi=-\mathbb{I}, \quad \psi \phi+C \psi=0, \quad \psi B+C^{2}=-\mathbb{I}, \quad \phi B+B C=0
\end{array}
$$

where $\mathbb{I}$ is the identity operator on the total space of $\Phi$.

Now, we give an example to understand quasi-hemi-slant conformal submersions better. 
Example 3.2. A map $\Phi: \mathbb{R}^{8} \longrightarrow \mathbb{R}^{4}$ is defined by

$$
\left(x_{1}, x_{2}, x_{3}, x_{4}, x_{5}, x_{6}, x_{7}, x_{8}\right) \longrightarrow\left(\frac{x_{1}+x_{3}}{\sqrt{2}}, x_{6}, x_{5}, x_{8}\right) .
$$

Then, we get the horizontal distribution

$$
\left(k e r \Phi_{*}\right)^{\perp}=\left\{Z_{1}=\frac{1}{\sqrt{2}}\left(\frac{\partial}{\partial x_{1}}+\frac{\partial}{\partial x_{3}}\right), Z_{2}=\frac{\partial}{\partial x_{6}}, Z_{3}=\frac{\partial}{\partial x_{5}}, Z_{4}=\frac{\partial}{\partial x_{8}}\right\},
$$

and the vertical distribution

$$
k e r \Phi_{*}=\left\{V_{1}=\frac{\partial}{\partial x_{2}}, V_{2}=\frac{\partial}{\partial x_{4}}, V_{3}=\frac{\partial}{\partial x_{7}}, V_{4}=\frac{\partial}{\partial x_{1}}-\frac{\partial}{\partial x_{3}}\right\} .
$$

Hence, using complex structure $J=\left(-x_{2}, x_{1},-x_{4}, x_{3},-x_{6}, x_{5},-x_{8}, x_{7}\right)$ of $\mathbb{R}^{8}$ on the distributions, we obtain

$$
\begin{array}{r}
J\left(Z_{1}\right)=\frac{1}{\sqrt{2}}\left(V_{1}+V_{2}\right), \quad J\left(Z_{2}\right)=-Z_{3}, \quad J\left(Z_{3}\right)=Z_{2}, \quad J\left(Z_{4}\right)=-V_{3}, \\
J\left(V_{1}\right)=-\frac{\sqrt{2}}{2} Z_{1}-\frac{1}{2} V_{4}, \quad J\left(V_{2}\right)=-\frac{\sqrt{2}}{2} Z_{1}+\frac{1}{2} V_{4}, \quad J\left(V_{3}\right)=Z_{4}, \quad J\left(V_{4}\right)=V_{1}-V_{2} .
\end{array}
$$

Therefore, we get $D=s p\left\{V_{4}\right\}, D_{\theta}=s p\left\{V_{1}, V_{2}\right\}, D_{\perp}=s p\left\{V_{3}\right\}, \mu=s p\left\{Z_{2}, Z_{3}\right\}, J\left(D_{\perp}\right)=s p\left\{Z_{4}\right\}$ and $\psi D_{\theta}=s p\left\{Z_{1}\right\}$. One can see that $\Phi$ is a quasi-hemi-slant conformal submersion with $\wedge=\lambda^{2}=1$ and quasi-hemi-slant angle $\theta=\frac{\pi}{2}$.

In the rest of this study, we assume that $\Phi:\left(M, g_{M}, J\right) \longrightarrow\left(N, g_{N}\right)$ is a quasi-hemi-slant conformal submersion from a Kähler manifold $\left(M, g_{M}, J\right)$ to a Riemannian manifold $\left(N, g_{N}\right)$. We have the following lemma which has the same proof for quasi-hemi-slant submersions.

Lemma 3.3. Let $\Phi:\left(M, g_{M}, J\right) \longrightarrow\left(N, g_{N}\right)$ be a slant submersion. Then,

$$
\begin{array}{r}
-\phi^{2} X=\cos ^{2} \theta X, \\
g_{M}(\phi X, \phi Y)=\cos ^{2} \theta g_{M}(X, Y), \\
g_{M}(\psi X, \psi Y)=\sin ^{2} \theta g_{M}(X, Y)
\end{array}
$$

for $X, Y \in \Gamma\left(D_{\theta}\right)[16]$.

Throughout this section, we give necessary and sufficient conditions to be integrability for distributions.

Theorem 3.4. Let $\Phi:\left(M, g_{M}, J\right) \longrightarrow\left(N, g_{N}\right)$ be a quasi-hemi-slant conformal submersion. Then, the distribution $D_{\theta}$ is integrable if and only if

$$
\begin{aligned}
g_{N}\left(\left(\nabla \Phi_{*}\right)\left(V_{2}, \phi \tilde{P} \xi\right), \Phi_{*}\left(\psi V_{1}\right)\right)-g_{N}\left(\left(\nabla \Phi_{*}\right)\left(V_{1}, \phi \tilde{P} \xi\right), \Phi_{*}\left(\psi V_{2}\right)\right)= & \lambda^{2}\left\{g_{M}\left(\hat{\nabla}_{V_{2}} \phi \tilde{P} \xi+T_{V_{2}} \psi \tilde{R} \xi, \phi V_{1}\right)-g_{M}\left(h \vec{\nabla}_{V_{1}} \psi \tilde{R} \xi, \psi V_{2}\right)\right. \\
& \left.+g_{M}\left(h \nabla_{V_{2}} \psi \tilde{R} \xi, \psi V_{1}\right)-g_{M}\left(\hat{\nabla}_{V_{1}} \phi \tilde{P} \xi+T_{V_{1}} \psi \tilde{R} \xi, \phi V_{2}\right)\right\}
\end{aligned}
$$

for $V_{1}, V_{2} \in \Gamma\left(D_{\theta}\right)$ and $\xi \in \Gamma\left(D \oplus D_{\perp}\right)$.

Proof. Since $M$ is a Kähler manifold, we have $g_{M}\left(\stackrel{M}{\nabla} V_{V_{1}} V_{2}, \xi\right)=-g_{M}\left(\stackrel{M}{\nabla_{V_{1}}} J \xi, J V_{2}\right)$ for $V_{1}, V_{2} \in \Gamma\left(D_{\theta}\right)$ and $\xi \in \Gamma\left(D \oplus D_{\perp}\right)$. So, we get from (2.6), (2.7), (3.3) and (3.4)

$$
\begin{aligned}
-g_{M}\left(\stackrel{M}{\nabla}_{V_{1}} J \xi, J V_{2}\right) & =-g_{M}\left(\stackrel{M}{\nabla_{V_{1}}} \phi \tilde{P} \xi+{\stackrel{M}{V_{1}}} \psi \tilde{R} \xi, \phi V_{2}+\psi V_{2}\right) \\
& =-g_{M}\left(\hat{\nabla}_{V_{1}} \phi \tilde{P} \xi+T_{V_{1}} \psi \tilde{R} \xi, \phi V_{2}\right)-g_{M}\left(T_{V_{1}} \phi \tilde{P} \xi+h \nabla_{V_{1}} \psi \tilde{R} \xi, \psi V_{2}\right) .
\end{aligned}
$$

Changing the roles of $V_{1}$ and $V_{2}$ in (3.14), we have second part of $g_{M}\left(\left[V_{1}, V_{2}\right], \xi\right)$. Hence, from (2.11) we obtain

$$
\begin{aligned}
g_{M}\left(\left[V_{1}, V_{2}\right], \xi\right)= & g_{M}\left(\hat{\nabla}_{V_{2}} \phi \tilde{P} \xi+T_{V_{2}} \psi \tilde{R} \xi, \phi V_{1}\right)-g_{M}\left(\hat{\nabla}_{V_{1}} \phi \tilde{P} \xi+T_{V_{1}} \psi \tilde{R} \xi, \phi V_{2}\right) \\
& +g_{M}\left(h \nabla_{V_{2}} \psi \tilde{R} \xi, \psi V_{1}\right)-g_{M}\left(h \nabla_{V_{1}} \psi \tilde{R} \xi, \psi V_{2}\right) \\
& +\frac{1}{\lambda^{2}}\left\{g_{N}\left(\left(\nabla \Phi_{*}\right)\left(V_{1}, \phi \tilde{P} \xi\right), \Phi_{*}\left(\psi V_{2}\right)\right)-g_{N}\left(\left(\nabla \Phi_{*}\right)\left(V_{2}, \phi \tilde{P} \xi\right), \Phi_{*}\left(\psi V_{1}\right)\right)\right\}
\end{aligned}
$$

The proof is completed from (3.15). 
In a similar way, we have the following theorem.

Theorem 3.5. Let $\Phi:\left(M, g_{M}, J\right) \longrightarrow\left(N, g_{N}\right)$ be a quasi-hemi-slant conformal submersion. Then, the distribution $D$ is integrable if and only if

$$
\tilde{P}\left(\hat{\nabla}_{U_{1}} \phi \tilde{Q} \xi+T_{U_{1}} \psi \xi\right)=0
$$

for $U_{1}, U_{2} \in \Gamma(D)$ and $\xi \in \Gamma\left(D_{\theta} \oplus D_{\perp}\right)$.

Proof. Using (2.2), (2.6), (2.7) and (3.5), we have

$$
\begin{aligned}
g_{M}\left(\stackrel{M}{\nabla}_{U_{1}} U_{2}, \xi\right) & =-g_{M}\left(\nabla_{U_{1}} \phi \tilde{Q} \xi+\psi \tilde{Q} \xi+\psi \tilde{R} \xi, J U_{2}\right) \\
& =-g_{M}\left(\hat{\nabla}_{U_{1}} \phi \tilde{Q} \xi+T_{U_{1}} \psi \tilde{Q} \xi+T_{U_{1}} \psi \tilde{R} \xi, J U_{2}\right)
\end{aligned}
$$

for $U_{1}, U_{2} \in \Gamma(D)$ and $\xi \in \Gamma\left(D_{\theta} \oplus D_{\perp}\right)$. Now, since $\psi(\tilde{Q} \xi+\tilde{R} \xi)=\psi \xi$ and from (3.16) we obtain

$$
g_{M}\left(\left[U_{1}, U_{2}\right], \xi\right)=g_{M}\left(\hat{\nabla}_{U_{2}} \phi \tilde{Q} \xi+T_{U_{2}} \psi \xi, J U_{1}\right)-g_{M}\left(\hat{\nabla}_{U_{1}} \phi \tilde{Q} \xi+T_{U_{1}} \psi \xi, J U_{2}\right)
$$

Since $D$ is an invariant distribution, we have $J U_{1}, J U_{2} \in \Gamma(D)$. Therefore, we obtain the proof from (3.17).

Here, integrability condition of the anti-invariant distribution $D_{\perp}$ is same as the condition for hemi-slant submersions in [20]. In addition, we know that the vertical distribution of a submersion is always integrable. Hence, we lastly give integrability condition for the horizontal distribution $\left(k e r \Phi_{*}\right)^{\perp}$.

Theorem 3.6. Let $\Phi:\left(M, g_{M}, J\right) \longrightarrow\left(N, g_{N}\right)$ be a quasi-hemi-slant conformal submersion. Then, the distribution $\left(\text { ker } \Phi_{*}\right)^{\perp}$ is integrable if and only if

$$
\begin{aligned}
g_{N}\left(\left(\nabla \Phi_{*}\right)\left(Z_{1}, B Z_{2}\right)-\left(\nabla \Phi_{*}\right)\left(Z_{2}, B Z_{1}\right)+\nabla_{Z_{2}}^{N} \Phi_{*}\left(C Z_{1}\right)-\nabla_{Z_{1}}^{\Phi} \Phi_{*}\left(C Z_{2}\right), \Phi_{*}(\psi \xi)\right) \\
=\lambda^{2} g_{M}\left(v \nabla_{Z_{1}} B Z_{2}-v \nabla_{Z_{2}} B Z_{1}, \phi \xi\right)+\lambda^{2} g_{M}\left(A_{Z_{1}} C Z_{2}-A_{Z_{2}} C Z_{1}, \phi \xi\right)-C Z_{2}(\ln \lambda) g_{M}\left(Z_{1}, \psi \xi\right) \\
\quad+\psi \xi(\ln \lambda) g_{M}\left(Z_{1}, C Z_{2}\right)+C Z_{1}(\ln \lambda) g_{M}\left(Z_{2}, \psi \xi\right)-\psi \xi(\ln \lambda) g_{M}\left(Z_{2}, C Z_{1}\right)
\end{aligned}
$$

for $Z_{1}, Z_{2} \in \Gamma\left(\left(\operatorname{ker} \Phi_{*}\right)^{\perp}\right)$ and $\xi \in \Gamma\left(k \operatorname{er} \Phi_{*}\right)$.

Proof. Firstly, from (2.8), (2.9), (3.4) and (3.8), we have

$$
g_{M}\left(\stackrel{M}{\nabla} Z_{1} Z_{2}, \xi\right)=g_{M}\left(A_{Z_{1}} B Z_{2}+h \stackrel{M}{\nabla_{Z_{1}}} C Z_{2}, \psi \xi\right)+g_{M}\left(\nu{\stackrel{M}{Z_{1}}}_{Z_{1}} B Z_{2}+A_{Z_{1}} C Z_{2}, \phi \xi\right)
$$

for $Z_{1}, Z_{2} \in \Gamma\left(\left(\operatorname{ker} \Phi_{*}\right)^{\perp}\right)$ and $\xi \in \Gamma\left(k \operatorname{er} \Phi_{*}\right)$. Now, changing the roles of $Z_{1}$ and $Z_{2}$ in (3.18), we get

$$
\begin{aligned}
g_{M}\left(\left[Z_{1}, Z_{2}\right], \xi\right)= & g_{M}\left(A_{Z_{1}} B Z_{2}+h \stackrel{M}{\nabla_{Z_{1}}} C Z_{2}-A_{Z_{2}} B Z_{1}-h \nabla_{Z_{2}} C Z_{1}, \psi \xi\right) \\
& +g_{M}\left(v \nabla_{Z_{1}} B Z_{2}+A_{Z_{1}} C Z_{2}-v \stackrel{M}{\nabla_{Z_{2}}} B Z_{1}-A_{Z_{2}} C Z_{1}, \phi \xi\right)
\end{aligned}
$$

Hence, using equations (2.3), (2.10), (2.12) in (3.19) and since $\mu$ is orthogonal to $\psi D_{\theta} \oplus J\left(D_{\perp}\right)$, we obtain

$$
\begin{aligned}
0= & g_{M}\left(\nu \nabla_{Z_{1}} B Z_{2}+A_{Z_{1}} C Z_{2}-\nu \nabla_{Z_{2}} B Z_{1}-A_{Z_{2}} C Z_{1}, \phi \xi\right)+\frac{1}{\lambda^{2}} g_{N}\left(\left(\nabla \Phi_{*}\right)\left(Z_{2}, B Z_{1}\right)-\left(\nabla \Phi_{*}\right)\left(Z_{1}, B Z_{2}\right), \Phi_{*}(\psi \xi)\right) \\
& +\frac{1}{\lambda^{2}} g_{N}\left(\nabla_{Z_{1}}^{\Phi} \Phi_{*}\left(C Z_{2}\right)-\nabla_{Z_{2}}^{\Phi} \Phi_{*}\left(C Z_{1}\right), \Phi_{*}(\psi \xi)\right)-C Z_{2}(\ln \lambda) g_{M}\left(Z_{1}, \psi \xi\right)+\psi \xi(\ln \lambda) g_{M}\left(Z_{1}, C Z_{2}\right) \\
& +C Z_{1}(\ln \lambda) g_{M}\left(Z_{2}, \psi \xi\right)-\psi \xi(\ln \lambda) g_{M}\left(Z_{2}, C Z_{1}\right) .
\end{aligned}
$$

One can see the proof from (3.20). 


\section{Totally Geodesicness on Distributions} $M$.

In this section, we present conditions for certain distributions and the map $\Phi$ to define totally geodesic foliations on

Theorem 4.1. Let $\Phi:\left(M, g_{M}, J\right) \longrightarrow\left(N, g_{N}\right)$ be a quasi-hemi-slant conformal submersion. Then, the distribution $D$ defines totally geodesic foliations on $M$ if and only if

i) $\lambda^{2} g_{M}\left(\hat{\nabla}_{U_{1}} J U_{2}, \phi \tilde{Q} \xi\right)=g_{N}\left(\left(\nabla \Phi_{*}\right)\left(U_{1}, J U_{2}\right), \Phi_{*}(\psi \xi)\right)$,

ii) $\lambda^{2} g_{M}\left(\hat{\nabla}_{U_{1}} J U_{2}, B Z\right)=g_{N}\left(\left(\nabla \Phi_{*}\right)\left(U_{1}, J U_{2}\right), \Phi_{*}(C Z)\right)$

are provided for $U_{1}, U_{2} \in \Gamma(D), \xi \in \Gamma\left(D_{\theta} \oplus D_{\perp}\right)$ and $Z \in \Gamma\left(\left(\operatorname{ker} \Phi_{*}\right)^{\perp}\right)$.

Proof. Firstly, from (2.6), (2.11) and (3.4) we have

$$
\begin{aligned}
g_{M}\left(\stackrel{M}{\nabla}_{U_{1}} U_{2}, \xi\right) & =g_{M}\left(T_{U_{1}} J U_{2}, \psi \xi\right)+g_{M}\left(\hat{\nabla}_{U_{1}} J U_{2}, \phi \xi\right) \\
& =-\frac{1}{\lambda^{2}} g_{N}\left(\left(\nabla \Phi_{*}\right)\left(U_{1}, J U_{2}\right), \Phi_{*}(\psi \xi)\right)+g_{M}\left(\hat{\nabla}_{U_{1}} J U_{2}, \phi \xi\right)
\end{aligned}
$$

for $U_{1}, U_{2} \in \Gamma(D)$ and $\xi \in \Gamma\left(D_{\theta} \oplus D_{\perp}\right)$. On the other hand, from (2.6), (2.11) and (3.8) we have

$$
\begin{aligned}
g_{M}\left(\stackrel{M}{\nabla}_{U_{1}} U_{2}, Z\right) & =g_{M}\left(T_{U_{1}} J U_{2}, C Z\right)+g_{M}\left(\hat{\nabla}_{U_{1}} J U_{2}, B Z\right) \\
& =-\frac{1}{\lambda^{2}} g_{N}\left(\left(\nabla \Phi_{*}\right)\left(U_{1}, J U_{2}\right), \Phi_{*}(C Z)\right)+g_{M}\left(\hat{\nabla}_{U_{1}} J U_{2}, B Z\right) .
\end{aligned}
$$

We obtain (i) and (ii) from (4.1) and (4.2), respectively.

Theorem 4.2. Let $\Phi:\left(M, g_{M}, J\right) \longrightarrow\left(N, g_{N}\right)$ be a quasi-hemi-slant conformal submersion. Then, the distribution $D_{\theta}$ defines totally geodesic foliations on $M$ if and only if

$$
\begin{aligned}
& \text { i) }-\lambda^{2}\left\{\cos ^{2} \theta g_{M}\left(\hat{\nabla}_{V_{1}} \tilde{Q} V_{2}, \xi\right)+g_{M}\left(h \nabla_{V_{1}} \psi \tilde{Q} V_{2}, \psi \tilde{R} \xi\right)\right\} \quad=\quad g_{N}\left(\left(\nabla \Phi_{*}\right)\left(V_{1}, \xi\right), \Phi_{*}\left(\psi \phi \tilde{Q} V_{2}\right)\right) \\
& +g_{N}\left(\left(\nabla \Phi_{*}\right)\left(V_{1}, \phi \tilde{P} \xi\right), \Phi_{*}\left(\psi \tilde{Q} V_{2}\right)\right) \text {, } \\
& \text { ii) } \quad \lambda^{2}\left\{g_{M}\left(h \stackrel{M}{\nabla}_{V_{1}} \psi \phi \tilde{Q} V_{2}, Z\right)+g_{M}\left(h \stackrel{M}{\nabla}_{V_{1}} \psi \tilde{Q} V_{2}, C Z\right)\right\}=\cos ^{2} \theta g_{N}\left(\left(\nabla \Phi_{*}\right)\left(V_{1}, \tilde{Q} V_{2}\right), \Phi_{*}(Z)\right) \\
& -g_{N}\left(\left(\nabla \Phi_{*}\right)\left(V_{1}, B Z\right), \Phi_{*}\left(\psi \tilde{Q} V_{2}\right)\right)
\end{aligned}
$$

are provided for $V_{1}, V_{2} \in \Gamma\left(D_{\theta}\right), \xi \in \Gamma\left(D \oplus D_{\perp}\right)$ and $Z \in \Gamma\left(\left(k e r \Phi_{*}\right)^{\perp}\right)$.

Proof. From equations (2.6), (2.7), (2.11), (3.11) and skew-symmetry properties of $T$ we have

$$
\begin{aligned}
& g_{M}\left(\stackrel{M}{\nabla}_{V_{1}} V_{2}, \xi\right)=\cos ^{2} \theta g_{M}\left(\stackrel{M}{\nabla}_{V_{1}} \tilde{Q} V_{2}, \xi\right)+g_{M}\left(T_{V_{1}} \psi \phi \tilde{Q} V_{2}, \xi\right)+g_{M}\left(T_{V_{1}} \psi \tilde{Q} V_{2}, \phi \tilde{P} \xi\right)+g_{M}\left(h \stackrel{M}{\nabla_{V_{1}}} \psi \tilde{Q} V_{2}, \psi \tilde{R} \xi\right) \\
& =\cos ^{2} \theta g_{M}\left(\hat{\nabla}_{V_{1}} \tilde{Q} V_{2}, \xi\right)-g_{M}\left(T_{V_{1}} \xi, \psi \phi \tilde{Q} V_{2}\right)-g_{M}\left(T_{V_{1}} \phi \tilde{P} \xi, \psi \tilde{Q} V_{2}\right)+g_{M}\left(h \vec{\nabla}_{V_{1}} \psi \tilde{Q} V_{2}, \psi \tilde{R} \xi\right) \\
& =\cos ^{2} \theta g_{M}\left(\hat{\nabla}_{V_{1}} \tilde{Q} V_{2}, \xi\right)+g_{M}\left(h{\stackrel{M}{V_{1}}} \psi \tilde{Q} V_{2}, \psi \tilde{R} \xi\right) \\
& +\frac{1}{\lambda^{2}} g_{N}\left(\left(\nabla \Phi_{*}\right)\left(V_{1}, \xi\right), \Phi_{*}\left(\psi \phi \tilde{Q} V_{2}\right)\right)+\frac{1}{\lambda^{2}} g_{N}\left(\left(\nabla \Phi_{*}\right)\left(V_{1}, \phi \tilde{P} \xi\right), \Phi_{*}\left(\psi \tilde{Q} V_{2}\right)\right)
\end{aligned}
$$

for $V_{1}, V_{2} \in \Gamma\left(D_{\theta}\right)$ and $\xi \in \Gamma\left(D \oplus D_{\perp}\right)$. In a similar way, from (3.8) we have

$$
\begin{aligned}
& g_{M}\left(\stackrel{M}{\nabla}_{V_{1}} V_{2}, Z\right)=\cos ^{2} \theta g_{M}\left(\stackrel{M}{\nabla} V_{V_{1}} \tilde{Q} V_{2}, Z\right)+g_{M}\left(h \stackrel{M}{\nabla}_{V_{1}} \psi \phi \tilde{Q} V_{2}, Z\right)-g_{M}\left(T_{V_{1}} B Z, \psi \tilde{Q} V_{2}\right)+g_{M}\left(h \stackrel{M}{\nabla_{V_{1}}} \psi \tilde{Q} V_{2}, C Z\right) \\
& =\cos ^{2} \theta g_{M}\left(T_{V_{1}} \tilde{Q} V_{2}, Z\right)+g_{M}\left(h{\stackrel{M}{V_{1}}}_{\psi} \psi \phi \tilde{Q} V_{2}, Z\right)+\frac{1}{\lambda^{2}} g_{N}\left(\left(\nabla \Phi_{*}\right)\left(V_{1}, B Z\right), \Phi_{*}\left(\psi \tilde{Q} V_{2}\right)\right)+g_{M}\left(h \nabla_{V_{1}} \psi \tilde{Q} V_{2}, C Z\right) \\
& =-\cos ^{2} \theta \frac{1}{\lambda^{2}} g_{N}\left(\left(\nabla \Phi_{*}\right)\left(V_{1}, \tilde{Q} V_{2}\right), \Phi_{*}(Z)\right)+g_{M}\left(h \nabla_{V_{1}}^{M} \psi \phi \tilde{Q} V_{2}, Z\right) \\
& +\frac{1}{\lambda^{2}} g_{N}\left(\left(\nabla \Phi_{*}\right)\left(V_{1}, B Z\right), \Phi_{*}\left(\psi \tilde{Q} V_{2}\right)\right)+g_{M}\left(h \stackrel{M}{V}_{V_{1}} \psi \tilde{Q} V_{2}, C Z\right)
\end{aligned}
$$

for $V_{1}, V_{2} \in \Gamma\left(D_{\theta}\right)$ and $Z \in \Gamma\left(\left(k e r \Phi_{*}\right)^{\perp}\right)$. We obtain (i) and (ii) from (4.3) and (4.4), respectively. 
Theorem 4.3. Let $\Phi:\left(M, g_{M}, J\right) \longrightarrow\left(N, g_{N}\right)$ be a quasi-hemi-slant conformal submersion. Then, the distribution $D_{\perp}$ defines totally geodesic foliations on $M$ if and only if

$$
\begin{gathered}
\text { i) }-\lambda^{2} g_{M}\left(h{\stackrel{M}{W_{1}}}_{W_{1}} J W_{2}, \psi \tilde{Q} \xi\right)=g_{N}\left(\left(\nabla \Phi_{*}\right)\left(W_{1}, \phi \xi\right), \Phi_{*}\left(J W_{2}\right)\right), \\
\text { ii) }-\lambda^{2} g_{M}\left(h \nabla_{W_{1}} C Z, J W_{2}\right)=g_{N}\left(\left(\nabla \Phi_{*}\right)\left(W_{1}, B Z\right), \Phi_{*}\left(J W_{2}\right)\right)
\end{gathered}
$$

are provided for $W_{1}, W_{2} \in \Gamma\left(D_{\perp}\right), \xi \in \Gamma\left(D \oplus D_{\theta}\right)$ and $Z \in \Gamma\left(\left(k e r \Phi_{*}\right)^{\perp}\right)$.

Proof. Since the distribution $D$ is invariant from (3.3) and (3.4) we have $J \xi=\phi \xi+\psi \tilde{Q} \xi$. So, we get using skewsymmetry properties of $T,(2.7)$ and (2.11)

$$
\begin{aligned}
g_{M}\left(\nabla_{W_{1}}^{M} W_{2}, \xi\right) & =g_{M}\left(T_{W_{1}} J W_{2}, \phi \xi\right)+g_{M}\left(h \nabla_{W_{1}} J W_{2}, \psi \tilde{Q} \xi\right) \\
& =-g_{M}\left(T_{W_{1}} \phi \xi, J W_{2}\right)+g_{M}\left(h{\stackrel{\nabla}{W_{1}}} J W_{2}, \psi \tilde{Q} \xi\right) \\
& =\frac{1}{\lambda^{2}} g_{N}\left(\left(\nabla \Phi_{*}\right)\left(W_{1}, \phi \xi\right), \Phi_{*}\left(J W_{2}\right)\right)+g_{M}\left(h \nabla_{W_{1}} J W_{2}, \psi \tilde{Q} \xi\right)
\end{aligned}
$$

for $W_{1}, W_{2} \in \Gamma\left(D_{\perp}\right)$ and $\xi \in \Gamma\left(D \oplus D_{\theta}\right)$. Similarly, from (2.6), (2.7), (3.4) and (3.8) we get

$$
\begin{aligned}
g_{M}\left(\nabla_{W_{1}}^{M} W_{2}, Z\right) & =-g_{M}\left(T_{W_{1}} B Z+h{\stackrel{M}{W_{1}}}_{1} C Z, J W_{2}\right) \\
& =\frac{1}{\lambda^{2}} g_{N}\left(\left(\nabla \Phi_{*}\right)\left(W_{1}, B Z\right), \Phi_{*}\left(J W_{2}\right)\right)+g_{M}\left(h \nabla_{W_{1}} C Z, J W_{2}\right)
\end{aligned}
$$

for $W_{1}, W_{2} \in \Gamma\left(D_{\perp}\right)$ and $Z \in \Gamma\left(\left(\operatorname{ker} \Phi_{*}\right)^{\perp}\right)$. We obtain (i) and (ii) from (4.5) and (4.6), respectively.

Theorem 4.4. Let $\Phi:\left(M, g_{M}, J\right) \longrightarrow\left(N, g_{N}\right)$ be a quasi-hemi-slant conformal submersion. Then, the vertical distribution $k e r \Phi_{*}$ defines totally geodesic foliations on $M$ if and only if

$$
\begin{aligned}
& \lambda^{2}\left\{g_{M}\left(h \nabla_{\xi_{1}} \psi \tilde{Q} \xi_{2}+T_{\xi_{1}} \psi \tilde{R} \xi_{2}, C Z\right)-g_{M}\left(h \nabla_{\xi_{1}} \psi \phi \tilde{Q} \xi_{2}, Z\right)+g_{M}\left(\hat{\nabla}_{\xi_{1}} \phi \tilde{P} \xi_{2}+T_{\xi_{1}} \psi \tilde{Q} \xi_{2}+v \stackrel{M}{M} \nabla_{\xi_{1}} \psi \tilde{R} \xi_{2}, B Z\right)\right\} \\
& =\cos ^{2} \theta g_{N}\left(\left(\nabla \Phi_{*}\right)\left(\xi_{1}, \tilde{Q} \xi_{2}\right), \Phi_{*}(Z)\right)+g_{N}\left(\left(\nabla \Phi_{*}\right)\left(\xi_{1}, \phi \tilde{P} \xi_{2}\right), \Phi_{*}(C Z)\right)
\end{aligned}
$$

is provided for $\xi_{1}, \xi_{2} \in \Gamma\left(\operatorname{ker} \Phi_{*}\right)$ and $Z \in \Gamma\left(\left(\operatorname{ker} \Phi_{*}\right)^{\perp}\right)$.

Proof. We calculate the case of $g_{M}\left(\nabla_{\xi_{1}} \xi_{2}, Z\right)=0$ for $\xi_{1}, \xi_{2} \in \Gamma\left(k e r \Phi_{*}\right)$ and $Z \in \Gamma\left(\left(k e r \Phi_{*}\right)^{\perp}\right)$. So, (2.6), (2.7) and (3.5) we have

$$
\begin{aligned}
g_{M}\left(\nabla_{\xi_{1}}^{M} \xi_{2}, Z\right)= & g_{M}\left(\nabla_{\xi_{1}} \phi \tilde{P} \xi_{2}+\phi \tilde{Q} \xi_{2}+\psi \tilde{Q} \xi_{2}+\psi \tilde{R} \xi_{2}, J Z\right) \\
= & g_{M}\left(T_{\xi_{1}} \phi \tilde{P} \xi_{2}+h \nabla_{\xi_{1}} \psi \tilde{Q} \xi_{2}+T_{\xi_{1}} \psi \tilde{R} \xi_{2}, C Z\right) \\
& +g_{M}\left(\hat{\nabla}_{\xi_{1}} \phi \tilde{P} \xi_{2}+T_{\xi_{1}} \psi \tilde{Q} \xi_{2}+\nu \stackrel{M}{\nabla_{\xi_{1}}} \psi \tilde{R} \xi_{2}, B Z\right) \\
& -g_{M}\left(\nabla_{\xi_{1}} \phi^{2} \tilde{Q} \xi_{2}+{ }_{\xi_{1}} \psi \phi \tilde{Q} \xi_{2}, Z\right) .
\end{aligned}
$$

Here, we use equations (2.11) and (3.11) in (4.7). Hence, we obtain

$$
\begin{aligned}
0= & g_{M}\left(T_{\xi_{1}} \phi \tilde{P} \xi_{2}+h \stackrel{M}{\nabla_{\xi_{1}}} \psi \tilde{Q} \xi_{2}+T_{\xi_{1}} \psi \tilde{R} \xi_{2}, C Z\right) \\
& +g_{M}\left(\hat{\nabla}_{\xi_{1}} \phi \tilde{P} \xi_{2}+T_{\xi_{1}} \psi \tilde{Q} \xi_{2}+v \nabla_{\xi_{1}} \psi \tilde{R} \xi_{2}, B Z\right) \\
& +\cos ^{2} \theta g_{M}\left(T_{\xi_{1}} \tilde{Q} \xi_{2}, Z\right)-g_{M}\left(h \nabla_{\xi_{1}} \psi \phi \tilde{Q} \xi_{2}, Z\right) \\
= & -\frac{1}{\lambda^{2}} g_{N}\left(\left(\nabla \Phi_{*}\right)\left(\xi_{1}, \phi \tilde{P} \xi_{2}\right), \Phi_{*}(C Z)\right)+g_{M}\left(h \nabla_{\xi_{1}} \psi \tilde{Q} \xi_{2}+T_{\xi_{1}} \psi \tilde{R} \xi_{2}, C Z\right) \\
& +g_{M}\left(\hat{\nabla}_{\xi_{1}} \phi \tilde{P} \xi_{2}+T_{\xi_{1}} \psi \tilde{Q} \xi_{2}+v \nabla_{\xi_{1}} \psi \tilde{R} \xi_{2}, B Z\right) \\
& -\cos ^{2} \theta \frac{1}{\lambda^{2}} g_{N}\left(\left(\nabla \Phi_{*}\right)\left(\xi_{1}, \tilde{Q} \xi_{2}\right), \Phi_{*}(Z)\right)-g_{M}\left(h \nabla_{\xi_{1}} \psi \phi \tilde{Q} \xi_{2}, Z\right)
\end{aligned}
$$

The proof is completed from (4.8). 
Theorem 4.5. Let $\Phi:\left(M, g_{M}, J\right) \longrightarrow\left(N, g_{N}\right)$ be a quasi-hemi-slant conformal submersion. Then, the horizontal distribution $\left(k e r \Phi_{*}\right)^{\perp}$ defines totally geodesic foliations on $M$ if and only if

$$
\begin{aligned}
\frac{1}{\lambda^{2}}\left\{g_{N}\left(\left(\nabla \Phi_{*}\right)\left(Z_{1}, B Z_{2}\right), \Phi_{*}(\psi \xi)\right)-g_{N}\left(\left(\nabla \Phi_{*}\right)\left(Z_{1}, \phi \xi\right), \Phi_{*}\left(C Z_{2}\right)\right)\right\}= & g_{M}\left(v \nabla_{Z_{1}} B Z_{2}, \phi \xi\right)+C Z_{2}(\ln \lambda) g_{M}\left(Z_{1}, \psi \xi\right) \\
& -\psi \xi(\ln \lambda) g_{M}\left(Z_{1}, C Z_{2}\right)
\end{aligned}
$$

is provided for $Z_{1}, Z_{2} \in \Gamma\left(\left(k e r \Phi_{*}\right)^{\perp}\right)$ and $\xi \in \Gamma\left(k e r \Phi_{*}\right)$.

Proof. Using equations (2.2), (2.8), (2.9) and (3.4), we get

$$
\begin{aligned}
g_{M}\left(\nabla_{Z_{1}} Z_{2}, \xi\right) & =g_{M}\left(\stackrel{M}{\nabla_{Z_{1}} B Z_{2}}+\stackrel{M}{\nabla_{Z_{1}}} C Z_{2}, \phi \xi+\psi \xi\right) \\
& =g_{M}\left(A_{Z_{1}} B Z_{2}+h{ }^{M} \nabla_{Z_{1}} C Z_{2}, \psi \xi\right)+g_{M}\left(\nu \nabla_{Z_{1}} B Z_{2}+A_{Z_{1}} C Z_{2}, \phi \xi\right)
\end{aligned}
$$

for $Z_{1}, Z_{2} \in \Gamma\left(\left(k e r \Phi_{*}\right)^{\perp}\right)$ and $\xi \in \Gamma\left(k e r \Phi_{*}\right)$. Here, we apply (2.10), (2.12), (3.8) to (4.9) and from skew-symmetric properties of $A$, we obtain

$$
\begin{aligned}
g_{M}\left(\stackrel{M}{\nabla} Z_{1} Z_{2}, \xi\right)= & -\frac{1}{\lambda^{2}} g_{N}\left(\left(\nabla \Phi_{*}\right)\left(Z_{1}, B Z_{2}\right), \Phi_{*}(\psi \xi)\right)+C Z_{2}(\ln \lambda) g_{M}\left(Z_{1}, \psi \xi\right)-\psi \xi(\ln \lambda) g_{M}\left(Z_{1}, C Z_{2}\right) \\
& +g_{M}\left(\nu{\stackrel{M}{Z_{1}}} B Z_{2}, \phi \xi\right)+\frac{1}{\lambda^{2}} g_{N}\left(\left(\nabla \Phi_{*}\right)\left(Z_{1}, \phi \xi\right), \Phi_{*}\left(C Z_{2}\right)\right) .
\end{aligned}
$$

The proof is completed from (4.10)

Note that, a horizontally conformal submersion $\Phi:\left(M, g_{M}, J\right) \longrightarrow\left(N, g_{N}\right)$ is said to be horizontally homothetic if the gradient of its dilation $\lambda$ is vertical, i.e., $h(\operatorname{grad} \lambda)=0$ at regular points [19]. Hence, we have the following.

Corollary 4.6. Let $\Phi:\left(M, g_{M}, J\right) \longrightarrow\left(N, g_{N}\right)$ be a quasi-hemi-slant conformal submersion. Then, the horizontal distribution $\left(k e r \Phi_{*}\right)^{\perp}$ defines totally geodesic foliations on $M$ if and only if

i) $\Phi$ is a horizontally homothetic map,

ii) $g_{N}\left(\left(\nabla \Phi_{*}\right)\left(Z_{1}, B Z_{2}\right), \Phi_{*}(\psi \xi)\right)-g_{N}\left(\left(\nabla \Phi_{*}\right)\left(Z_{1}, \phi \xi\right), \Phi_{*}\left(C Z_{2}\right)\right)=\lambda^{2} g_{M}\left(\nu \nabla_{Z_{1}}^{M} B Z_{2}, \phi \xi\right)$

are provided for $Z_{1}, Z_{2} \in \Gamma\left(\left(\operatorname{ker} \Phi_{*}\right)^{\perp}\right)$ and $\xi \in \Gamma\left(\operatorname{ker} \Phi_{*}\right)$.

Proof. Because of $\Phi$ defines totally geodesic foliations on $M$, we have (4.10). Suppose that $\Phi$ is a horizontally homothetic map, we have from (4.10)

$$
0=C Z_{2}(\ln \lambda) g_{M}\left(Z_{1}, \psi \xi\right)-\psi \xi(\ln \lambda) g_{M}\left(Z_{1}, C Z_{2}\right)
$$

for $Z_{1}, Z_{2} \in \Gamma\left(\left(k e r \Phi_{*}\right)^{\perp}\right)$ and $\xi \in \Gamma\left(k e r \Phi_{*}\right)$. Here, if we take $Z_{1}=\psi \xi$ in (4.11) we get

$$
0=C Z_{2}(\ln \lambda) g_{M}(\psi \xi, \psi \xi)
$$

In (4.12), we get $0=C Z_{2}(\ln \lambda)$ and it means $\lambda$ is a constant on $\mu$. Similarly, if we take $Z_{1}=C Z_{2}$ in (4.11) we get

$$
0=-\psi \xi(\ln \lambda) g_{M}\left(C Z_{2}, C Z_{2}\right)
$$

In (4.13), we get $0=\psi \xi(\ln \lambda)$ and it means $\lambda$ is a constant on $\psi D_{\theta} \oplus J\left(D_{\perp}\right)$. Therefore, from (4.12) and (4.13) we say that $\lambda$ is a constant on horizontal distribution. So, (i) is satisfied. Now, if (i) is satisfied in (4.10), we obtain

$$
0=-\frac{1}{\lambda^{2}} g_{N}\left(\left(\nabla \Phi_{*}\right)\left(Z_{1}, B Z_{2}\right), \Phi_{*}(\psi \xi)\right)+g_{M}\left(v \nabla_{Z_{1}} B Z_{2}, \phi \xi\right)+\frac{1}{\lambda^{2}} g_{N}\left(\left(\nabla \Phi_{*}\right)\left(Z_{1}, \phi \xi\right), \Phi_{*}\left(C Z_{2}\right)\right) .
$$

From (4.14), (ii) is satisfied. The proof is completed.

A horizontally conformal submersion $\Phi:\left(M, g_{M}, J\right) \longrightarrow\left(N, g_{N}\right)$ is said to be totally geodesic if second fundamental form of the map $\left(\nabla \Phi_{*}\right)(X, Y)=0$ for $X, Y \in \Gamma(T M)$ [4]. Hence, we have the next theorem.

Theorem 4.7. Let $\Phi:\left(M, g_{M}, J\right) \longrightarrow\left(N, g_{N}\right)$ be a quasi-hemi-slant conformal submersion. Then, the map $\Phi$ is totally geodesic if and only if

i) $\cos ^{2} \theta T_{\xi_{1}} \tilde{Q} \xi_{2}=h \stackrel{M}{\nabla_{\xi_{1}}} \psi \phi \tilde{Q} \xi_{2}+C\left\{T_{\xi_{1}} \phi \tilde{P} \xi_{2}+h \stackrel{M}{h \nabla_{\xi_{1}}} \psi \xi_{2}\right\}+\psi\left\{\hat{\nabla}_{\xi_{1}} \phi \tilde{P} \xi_{2}+T_{\xi_{1}} \psi \xi_{2}\right\}$,

ii) $0=C\left\{A_{Z_{1}} \phi \xi_{1}+h \stackrel{M}{\nabla_{Z_{1}}} \psi \xi_{1}\right\}+\psi\left\{v \stackrel{M}{\nabla_{Z_{1}}} \phi \xi_{1}+A_{Z_{1}} \psi \xi_{1}\right\}$ 
iii) $\Phi$ is a horizontally homothetic map

are provided for $Z_{1}, Z_{2} \in \Gamma\left(\left(k e r \Phi_{*}\right)^{\perp}\right)$ and $\xi_{1}, \xi_{2} \in \Gamma\left(k e r \Phi_{*}\right)$.

Proof. Firstly, we examine $\left(\nabla \Phi_{*}\right)\left(\xi_{1}, \xi_{2}\right)$ for $\xi_{1}, \xi_{2} \in \Gamma\left(k e r \Phi_{*}\right)$. Because of $\psi \tilde{Q} \xi_{2}+\psi \tilde{R} \xi_{2}=\psi \xi_{2}$ we have from (2.2), (2.3) and (3.5)

$$
\left(\nabla \Phi_{*}\right)\left(\xi_{1}, \xi_{2}\right)=\Phi_{*}\left(J \nabla_{\xi_{1}} \phi \tilde{P} \xi_{2}+\phi \tilde{Q} \xi_{2}+\psi \xi_{2}\right)
$$

for $\xi_{1}, \xi_{2} \in \Gamma\left(k e r \Phi_{*}\right)$. Then, using equations (2.6), (2.7) and (3.11) have

$$
\begin{aligned}
\left(\nabla \Phi_{*}\right)\left(\xi_{1}, \xi_{2}\right)= & \Phi_{*}\left(J T_{\xi_{1}} \phi \tilde{P} \xi_{2}+J \hat{\nabla}_{\xi_{1}} \phi \tilde{P} \xi_{2}\right) \\
& +\Phi_{*}\left(\stackrel{M}{\nabla_{\xi_{1}}} \phi^{2} \tilde{Q} \xi_{2}+\stackrel{M}{\nabla_{\xi_{1}}} \psi \phi \tilde{Q} \xi_{2}\right) \\
& +\Phi_{*}\left(J T_{\xi_{1}} \psi \xi_{2}+J h \vec{M}_{\xi_{1}} \psi \xi_{2}\right) \\
= & \Phi_{*}\left(C T_{\xi_{1}} \phi \tilde{P} \xi_{2}+\psi \hat{\nabla}_{\xi_{1}} \phi \tilde{P} \xi_{2}\right) \\
& -\cos ^{2} \theta \Phi_{*}\left(\stackrel{M}{\nabla_{\xi_{1}}} \tilde{Q} \xi_{2}\right)+\Phi_{*}\left(h \nabla_{\xi_{1}}^{M} \psi \phi \tilde{Q} \xi_{2}\right) \\
& +\Phi_{*}\left(\psi T_{\xi_{1}} \psi \xi_{2}+C h \nabla_{\xi_{1}} \psi \xi_{2}\right) \\
= & \Phi_{*}\left(C\left\{T_{\xi_{1}} \phi \tilde{P} \xi_{2}+h{\stackrel{M}{\xi_{1}}}_{\xi_{1}} \psi \xi_{2}\right\}+\psi\left\{\hat{\nabla}_{\xi_{1}} \phi \tilde{P} \xi_{2}+T_{\xi_{1}} \psi \xi_{2}\right\}\right) \\
& -\cos ^{2} \theta \Phi_{*}\left(T_{\xi_{1}} \tilde{Q} \xi_{2}\right)+\Phi_{*}\left(h \nabla_{\xi_{1}} \psi \phi \tilde{Q} \xi_{2}\right)
\end{aligned}
$$

We obtain (i) from (4.15). Second fundamental form of a map is symmetric. So, we have $\left(\nabla \Phi_{*}\right)\left(\xi_{1}, Z_{1}\right)=\left(\nabla \Phi_{*}\right)\left(Z_{1}, \xi_{1}\right)$ for $Z_{1} \in \Gamma\left(\left(k e r \Phi_{*}\right)^{\perp}\right)$ and $\xi_{1} \in \Gamma\left(k e r \Phi_{*}\right)$. From (2.3), (2.8), (2.9) and (3.4) we obtain

$$
\begin{aligned}
\left(\nabla \Phi_{*}\right)\left(Z_{1}, \xi_{1}\right) & =\stackrel{M}{M} \stackrel{M}{ } \Phi_{*}\left(J \nabla_{Z_{1}} \phi \xi_{1}+J \nabla_{Z_{1}} \psi \xi_{1}\right) \\
& =\Phi_{*}\left(J A_{Z_{1}} \phi \xi_{1}+J v \stackrel{M}{\nabla_{Z_{1}} \phi \xi_{1}}\right)+\Phi_{*}\left(J A_{Z_{1}} \psi \xi_{1}+J h{\stackrel{M}{Z_{1}}}_{\xi_{1}}\right) \\
& =\Phi_{*}\left(C A_{Z_{1}} \phi \xi_{1}+\psi v{\stackrel{M}{Z_{1}}} \phi \xi_{1}\right)+\Phi_{*}\left(\psi A_{Z_{1}} \psi \xi_{1}+C h \nabla_{Z_{1}} \psi \xi_{1}\right) .
\end{aligned}
$$

We obtain (ii) from (4.16). Lastly, from (2.10) we have

$$
\left(\nabla \Phi_{*}\right)\left(Z_{1}, Z_{2}\right)=Z_{1}(\ln \lambda) \Phi_{*}\left(Z_{2}\right)+Z_{2}(\ln \lambda) \Phi_{*}\left(Z_{1}\right)-g_{M}\left(Z_{1}, Z_{2}\right) \Phi_{*}(\operatorname{grad}(\ln \lambda))
$$

for $Z_{1}, Z_{2} \in \Gamma\left(\left(k e r \Phi_{*}\right)^{\perp}\right)$. For $Z_{1}$ in (4.17) we obtain

$$
\begin{aligned}
0 & =Z_{2}(\ln \lambda) g_{N}\left(\Phi_{*}\left(Z_{1}\right), \Phi_{*}\left(Z_{1}\right)\right) \\
0 & =\lambda^{2} Z_{2}(\ln \lambda) g_{M}\left(Z_{1}, Z_{1}\right) .
\end{aligned}
$$

In (4.18), we get $Z_{2}(\ln \lambda)=0$. It means $\lambda$ is a constant on horizontal distribution. So, the map is horizontally homothetic. (iii) is satisfied. The proof is completed.

\section{CONFLiCTS OF INTEREST}

The author declares that there are no conflicts of interest regarding the publication of this article.

\section{REFERENCES}

[1] Akyol, M.A., Conformal semi-slant submersions, Int. J. Geom. Methods Mod. Phys., 14(7)(2017), 1750114.

[2] Akyol, M.A., Şahin, B., Conformal anti-invariant submersions from almost Hermitian manifolds, Turk. J. Math., 40(2016), 43-70.

[3] Akyol, M.A., Şahin, B., Conformal semi-invariant submersions, Commun. Contemp. Math., 19(2)(2017), 1650011.

[4] Akyol, M.A., Şahin, B., Conformal slant submersions, Hacet. J. Math. Stat., 48(1)(2019), 28-44.

[5] Baird, P., Wood, J.C., Harmonic Morphism between Riemannian Manifolds, Clarendon Press, Oxford, NY, 2003.

[6] Falcitelli, M., Ianus, S., Pastore, A. M., Riemannian Submersions and Related Topics, World Scientific, River Edge, NJ, 2004.

[7] Gray, A., Pseudo-Riemannian almost product manifolds and submersions, J. Math. Mech., 16(7)(1967), 715-737.

[8] Kumar, S., Kumar, S., Pandey, S., Prasad, R., Conformal hemi-slant submersions from almost Hermitian manifolds, Commun. Korean Math. Soc., 35(3)(2020), 999-1018.

[9] Longwap, S., Massamba, F., Homti, N. E., On quasi-hemi-slant Riemannian submersion, J. Adv. Math. Comput. Sci., 34(1)(2019), 1-14. 
[10] Miao, J., Wang, Y., Gu, X., Yau, S. T., Optimal global conformal surface parametrization for visualization, Commun. Inf. Syst., 4(2)(2005), 117-134.

[11] Nore, T., Second fundamental form of a map, Ann. Mat. Pur. and Appl., 146(1986), 281-310.

[12] O'Neill, B., The fundamental equations of a submersion, Mich. Math. J., 13(4)(1966), 459-469.

[13] Park, K.S., Prasad, R., Semi-slant submersions, Bull. Korean Math. Soc., 50(3)(2013), 951-962.

[14] Şahin, B., Horizontally conformal submersions of CR-submanifolds, Kodai Math. J., 31(1)(2008), 46-53.

[15] Şahin, B., Anti-invariant Riemannian submersions from almost Hermitian manifolds, Cent. Eur. J. Math., 8(3)(2010), $437-447$.

[16] Şahin, B., Slant submersions from almost Hermitian manifolds, Bull. Math. Soc. Sci. Math. Roumanie (N.S.), 54(102)(1)(2011), 93-105.

[17] Şahin, B., Semi-invariant submersions from almost Hermitian manifolds, Can. Math. Bull., 56(1)(2013), $173-183$.

[18] Şahin, B., Riemannian submersions from almost Hermitian manifolds, Taiwan. J. Math., 17(2)(2013), 629-659.

[19] Şahin, B., Riemannian Submersions, Riemannian Maps in Hermitian Geometry, and Their Applications, Elsevier, London, 2017.

[20] Taştan, H.M., Şahin, B., Yanan, Ş., Hemi-slant submersions, Mediterr. J. Math., 13(2016), 2171-2184.

[21] Wang, Y., Gu, X., Yau, S.T., Volumetric harmonic map, Commun. Inf. Syst., 3(3)(2003), 191-202.

[22] Wang, Y., Gu, X., Chan, T.F., Thompson, P.M., Yau, S.T., Brain surface conformal parametrization with the Ricci flow, Proceeding of the IEEE International Symposium on Biomedical Imaging-from Nano to Macro (ISBI), (2007), 1312-1315.

[23] Watson, B., Almost Hermitian submersions, J. Differential Geom., 11(1)(1976), 147-165.

[24] Yano, K., Kon, M., Structures on Manifolds, Ser. Pure Math. World Scientific, Singapore, 1984. 